ORIGINAL ARTICLE

\title{
Comparison of three types of full-body compression garments on throwing and repeat-sprint performance in cricket players
}

\author{
Rob Duffield, Marc Portus
}

Br J Sports Med 2007;41:409-414. doi: 10.1136/bjsm.2006.033753

See end of article for authors' affiliations

Correspondence to Dr R Duffield, School of Human Movement, Charles Stuart University, Panorama Ave, Bathurst, NSW 2795, Australia; rdufffield@csu. edu.au

Accepted 7 February 2007 Published Online First 5 March 2007

\begin{abstract}
Objective: To compare the effects of three types of full-body compression garments (Skins, Adidas and Under Armour) on repeat-sprint and throwing performance in cricket players.

Methods: Following familiarisation, 10 male cricket players performed four randomised exercise sessions (3 garments and a control). Each session involved a 30 min repeat-sprint exercise protocol comprising $20 \mathrm{~m}$ sprints every minute, separated by submaximal exercise. Throwing tests included a pre-exercise and a postexercise maximal distance test and accuracy throwing tests. During each session, measures of heart rate, skin temperature, change in body mass, rate of perceived exertion and perceived muscle soreness were recorded. Capillary blood samples were analysed before and after exercise for lactate, $\mathrm{pH}, \mathrm{O}_{2}$ saturation and $\mathrm{O}_{2}$ partial pressure, and $24 \mathrm{~h}$ after exercise for creatine kinase (CK). Ratings of perceived muscle soreness were also obtained $24 \mathrm{~h}$ after exercise.

Results: No significant differences ( $p>0.05)$ were evident in repeat-sprint performance $(10 \mathrm{~m}, 20 \mathrm{~m}$ time or total submaximal distance covered) or throwing performance (maximum distance or accuracy). No significant differences ( $p>0.05$ ) were observed in heart rate, body mass change or blood measures during exercise. Significant differences $(p<0.05)$ were observed by way of higher mean skin temperature, lower 24 h postexercise $\mathrm{CK}$ values and lower $24 \mathrm{~h}$ postexercise ratings of muscle soreness when wearing compression garments. Analysis between respective brands of compression garments revealed no statistical differences ( $p>0.05)$.

Conclusions: No benefit was noted when wearing compression garments for repeat-sprint or throwing performance; however, the use of the garments as a recovery tool, when worn after exercise, may be beneficial to reduce postexercise trauma and perceived muscle soreness.
\end{abstract}

C ompression garments are elastic, body-moulded suits with an engineered compression gradient that can be worn as an upper-, lower- or full-body piece. Compression garments and elastic stockings have long been used in medicine to assist with venous return and reduce peripheral swelling in vascular patients. ${ }^{12}$ Relatively recently, commercially available compression garments have been proposed to provide performance benefits to athletes. ${ }^{3}$ These garments, worn during training and competition to aid performance and after exercise to speed recovery, are suggested to improve peripheral circulation and venous return, ${ }^{45}$ improve clearance of blood lactate $\left[\mathrm{La}^{-}\right],{ }^{6}$ reduce muscle oscillation ${ }^{7}$ and improve clearance of markers of muscle damage such as creatine kinase (CK). ${ }^{8}$

Of late, there has been an increase in the popularity of the use of compression garments across a range of sports, particularly among cricket players. Although a significant body of research evidence exists describing the role of compression garments in vascular distribution in diseased patients, less evidence exists for athletic sports performance. To date, only a small body of research supports the notion that the garments may provide some benefit to sports performance ${ }^{7}$ or aid recovery from exercise. ${ }^{8}$ Currently, several companies have garments for sale, with little performance-based evidence available to support their use or compare between brands for superior ergogenic benefits. In particular, there is currently no research on the effect of compression garments in improving exercise performance during high-intensity, intermittent activity such as that observed during repeat-sprint sports. Further, as previous studies have reported the improvement of performance in singular explosive movements such as a vertical jump, ${ }^{9}$ there is potential to transfer these benefits to improve explosive actions involved in cricket, including sprinting and throwing, as garments are often worn during games.
Therefore, the aim of this study was to compare the effects of three different types of full-body compression garments (Skins, Adidas and Under Armour) and a control condition (no compression garment) on performance in intermittent, repeat-sprint and throwing performance in cricket players.

\section{METHODS \\ Participants}

A total of 10 physically fit, male, club-level cricket players with mean (SD) age 22.1 (1.1) years, height 185.2 (6.5) $\mathrm{cm}$ and body mass $84.65(5.90) \mathrm{kg}$ were recruited for this study. All participants gave verbal and written consent to engage in all testing procedures, and ethical approval was granted by the institutional ethics committee.

\section{Overview}

Participants performed five testing sessions at the same time of the day, separated by $72-96 \mathrm{~h}$, and were required to abstain from ingestion of alcohol, caffeine and food substances $3 \mathrm{~h}$ before testing. All testing was conducted on an open (sheltered) $50 \mathrm{~m}$ synthetic track in a partially enclosed, biomechanics laboratory under cool environmental conditions (15 (3) ${ }^{\circ} \mathrm{C}$ ). Initial testing sessions familiarised participants with all measures and procedures involved in the testing protocol, while the remaining four sessions were identical, with the exception of the compression garments. The four sessions were

Abbreviations: AT, accuracy throwing; CK, creatine kinase; DT, distance throwing; ES, effect size; $H R$, heart rate; $H S D$, honest significant difference; $\left[\mathrm{La}^{-}\right]$, blood lactate; $\mathrm{MS}_{\mathrm{A}}$, muscle soreness for arm; $M S_{\mathrm{L}}$, muscle soreness for leg; $\mathrm{PO}_{2}$, partial pressure of oxygen; RPE, rating of perceived exertion; $\mathrm{sO}_{2}$, oxygen saturation of haemoglobin; $\mathrm{T}_{\text {sk, }}$, mean skin temperature; $\mathrm{UA}$, Under Armour; $\mathrm{VO}_{2 \text { max }}$, maximum amount of of oxygen in millilitres, one can use in 1 minute per kilogram of body weight 
Table 1 Mean (SD) $10 \mathrm{~m}$ time, total $10 \mathrm{~m}$ sprint time, \% decline in $10 \mathrm{~m}$ sprint time, $20 \mathrm{~m}$ sprint time, total $20 \mathrm{~m}$ sprint time, \% decline in $20 \mathrm{~m}$ sprint time and total distance covered for Skins, Adidas, Under Armour and control conditions

\begin{tabular}{lllll}
\hline & Skins & Adidas & UA & Control \\
\hline $10 \mathrm{~m}(\mathrm{~s})$ & $2.00(0.09)$ & $1.99(0.08)$ & $1.99(0.09)$ & $2.01(0.06)$ \\
$10 \mathrm{~m}$ total $(\mathrm{s})$ & $54.06(2.53)$ & $53.99(2.12)$ & $53.84(2.61)$ & $54.38(1.72)$ \\
$10 \mathrm{~m}$ decline $(\%)$ & $6.4(2.3)$ & $5.5(2.7)$ & $6.0(1.9)$ & $5.9(2.4)$ \\
$20 \mathrm{~m}(\mathrm{~s})$ & $3.42(0.16)$ & $3.42(0.14)$ & $3.40(0.15)$ & $3.48(0.08)$ \\
$20 \mathrm{~m}$ total $(\mathrm{s})$ & $92.35(4.27)$ & $92.47(3.80)$ & $92.06(4.13)$ & $93.98(2.05)$ \\
$20 \mathrm{~m}$ decline (\%) & $5.4(2.9)$ & $5.2(2.0)$ & $5.9(2.0)$ & $6.0(2.3)$ \\
Total distance $(\mathrm{m})$ & $3488.4(197.4)$ & $3484.4(176.4)$ & $3517.8(367.3)$ & $3370.7(239.8)$ \\
\hline UA, Under Armour. & & & & \\
\hline
\end{tabular}

randomised and included either no compression garment (control), a full-body Skins garment (Skins, Sydney, New South Wales, Australia), a full-body Under Armour (UA) garment (Under Armour, Baltimore, Maryland, USA) or a full-body Adidas garment (Adidas, Herzogenaurach, Germany). All compression garments were worn throughout the duration of the testing session (excluding nude mass measurement) and during the $24 \mathrm{~h}$ after exercise, during which participants abstained from exercise. Compression garments were fitted to participants on the basis of the respective company guidelines involving measures of height, weight and girth circumferences.

\section{Exercise protocol}

Following pre-exercise measures, participants performed a 3 min warm-up on a cycle ergometer (818E, Monark, Vansbro, Sweden) at $70 \mathrm{~W}$, followed by $3 \times 40 \mathrm{~m}$ runs of increasing speed, stretching of the upper and lower body, and a 5 min graduated throwing routine (progressive increase in throw distance and velocity). Following the warm-up, participants performed a maximal distance throwing (DT) test of five maximal cricket ball throws, each separated by $20 \mathrm{~s}$ recovery. Participants then performed an accuracy throwing (AT) test consisting of $2 \times 10,2 \times 20$ and $2 \times 30 \mathrm{~m}$ throws at a customdesigned target (Australian Institute of Sport, Canberra, Australia), with an emphasis on accuracy and speed. The target consisted of a large vinyl screen with a central diagram of fullsized wickets, surrounded by zones allocated with a numerical score (the score decreases further from the wickets). Performance on the AT test was measured by both speed to complete all six throws and a total score based on the accuracy of each throw.

Following the AT test, participants performed a $30 \mathrm{~min}$ intermittent, repeat-sprint exercise protocol consisting of a $20 \mathrm{~m}$ sprint every minute, separated by $45 \mathrm{~s}$ of submaximal exercise. The submaximal (recovery) exercise between sprints consisted of performing shuttle runs over $20 \mathrm{~m}$ for the remainder of the minute (until $10 \mathrm{~s}$ to go-approximately
45 s) before moving to the start line to complete the next sprint (start of next minute). The shuttle runs were classified as "hard", requiring participants to cover as much distance as possible in the allocated time; "moderate", which involved participants jogging at a self-selected pace; and "easy", which consisted of walking. Submaximal exercise modes were rotated following each sprint, with one recovery mode performed during the remainder of the minute, with 10 efforts made for each respective submaximal run. Performance was determined from $10 \mathrm{~m}$ and $20 \mathrm{~m}$ sprint time (speed light infra-red timing system, Swift, Lismore, New South Wales, Australia), \% decline in sprint time ((total time/(fastest time $\cdot$ sprint $\mathrm{n}) \cdot 100))$ and distance covered during the submaximal exercise bouts (SP10 GPS, GPSports, Canberra, Australia). At 10, 20 and 30 min of the exercise protocol, participants performed the AT test, and, following the final AT test, they completed a repeat of the maximal DT test.

\section{Measures}

Before and after each testing session, nude mass was measured on a set of calibrated scales (HW 100K, A \& D, Tokyo, Japan) to estimate changes in body mass due to sweat loss. Heart rate was measured (F1, Polar Electo Oy, Kempele, Finland) before exercise and every $5 \mathrm{~min}$ throughout the exercise protocol. Skin temperature was measured at three sites, including the sternum, forearm and calf (Monotherm 4070, Mallinkrodt, St Louis, Missouri, USA), before exercise, and every 10 min during and after exercise, to calculate the mean skin temperature $\left(\mathrm{T}_{\mathrm{sk}}\right)$ using the equation of Burton ${ }^{10}$ as previously used by Adams et al. ${ }^{11}$ Rating of perceived exertion (RPE) was obtained before exercise, at 10 min intervals during and after exercise, whereas the rating of perceived muscle soreness was obtained before and after exercise and $24 \mathrm{~h}$ after exercise for arm $\left(\mathrm{MS}_{\mathrm{A}}\right)$ and leg $\left(\mathrm{MS}_{\mathrm{L}}\right)$ muscles, respectively. Before and after exercise, $100 \mu \mathrm{l}$ of capillary blood was sampled from a finger of the non-throwing arm for analysis of $\left[\mathrm{La}^{-}\right], \mathrm{pH}$, oxygen saturation of haemoglobin $\left(\mathrm{sO}_{2}\right)$ and partial pressure of oxygen $\left(\mathrm{pO}_{2}\right) \quad(\mathrm{ABL} 825$

Table 2 Mean (SD) premaximal and postmaximal distance throw (DT), pretotal and post-total distance thrown (total of five throws), \% decline within pre-DT and post-DT, and overall decline in DT from pretest to post-test for Skins, Adidas, Under Armour and control conditions

\begin{tabular}{lllll}
\hline & Skins & Adidas & UA & Control \\
\hline DT pre $(\mathrm{m})$ & $63.2(11.7)$ & $61.7(11.7)$ & $62.5(9.9)$ & $62.2(8.2)$ \\
DT pretotal $(\mathrm{m})$ & $316.2(58.7)$ & $308.7(58.3$ & $312.6(49.6)$ & $311.3(41.0)$ \\
DT predecline (\%) & $4.3(2.6)$ & $6.3(3.5)$ & $4.9(2.0)$ & $5.4(0.9)$ \\
DT post $(\mathrm{m})$ & $59.6(12.0)$ & $60.6(10.7)$ & $58.7(9.4)$ & $59.2(9.6)$ \\
DT posttotal (m) & $297.9(60.1)$ & $303.1(53.9)$ & $293.8(46.9)$ & $296.3(50.4)$ \\
DT postdecline (\%) & $5.0(2.4)$ & $7.8(3.4)$ & $7.4(3.9)$ & $6.0(2.7)$ \\
Total decline (\%) & $6.2(2.4)$ & $3.9(2.2)^{*}$ & $6.4(2.8)$ & $5.1(2.9)$ \\
\hline
\end{tabular}

DT, distance throw; UA, Under Armour. *Large effect size ( $d>0.8)$. 

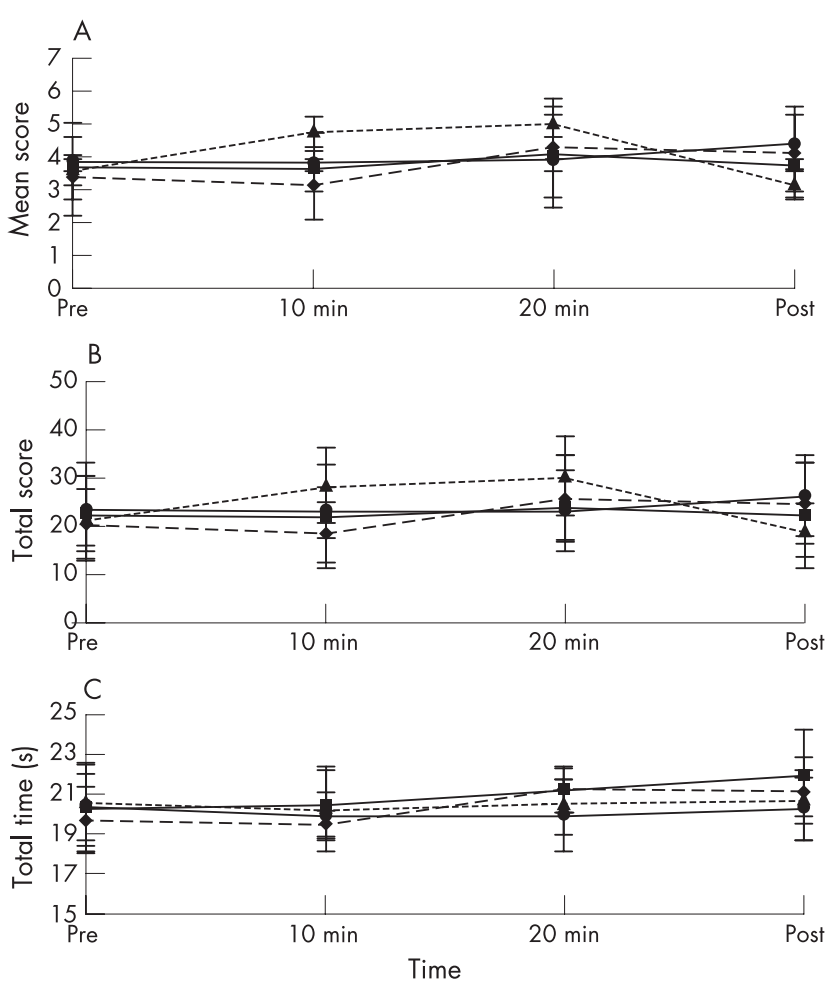

- Skins $\rightarrow$ Adidas $--\Delta$-UA $\rightarrow$-Control

Figure 1 (A) Mean individual throw score, (B) total score and (C) time to complete all throws for the accuracy throw test $(2 \times 10 \mathrm{~m}, 2 \times 20 \mathrm{~m}$ and $2 \times 30 \mathrm{~m}$ ) for all conditions. UA, Under Armour.

Radiometer, Copenhagen, Denmark). Finally, participants wore the garments for $24 \mathrm{~h}$ after exercise, at which point a $60 \mu \mathrm{l}$ sample of capillary blood was collected to measure CK as a marker of muscle damage. Blood samples were centrifuged to separate blood serum and analysed for CK (Dimension Xpand spectrophotometer, Dade Bearing, Newark, Delaware, USA).

\section{Statistical analyses}

A repeated-measures (condition $\times$ time) analysis of variance was used to determine significant differences between the respective conditions (Skins, Adidas, UA and control). Post hoc Tukey's honest significant difference (HSD) tests were used to determine individual significant differences. Significance was set a priori at $\mathrm{p}=0.05$. Effect sizes (ESs) (Cohen's d) were calculated to analyse potential trends in the data comparing respective compression garments with the control condition. An ES of $<0.2$ was classified as "trivial", 0.2-0.4 as "small", 0.40.7 as "moderate" and $>0.8$ as "large" effect.

\section{RESULTS}

Table 1 presents the exercise performance measures as mean (SD) 10 and $20 \mathrm{~m}$ sprint times, total 10 and $20 \mathrm{~m}$ sprint time, and $\%$ decline in 10 and $20 \mathrm{~m}$ sprint times. The total distance covered during the recovery bouts is also shown. Analysis indicated no significant differences $(\mathrm{p}>0.05)$ and small ES between conditions (Skins, Adidas, UA or control) for mean and total $10 \mathrm{~m}$ or $20 \mathrm{~m}$ sprint time or \% decline in $10 \mathrm{~m}$ or $20 \mathrm{~m}$ sprint time and total submaximal distance covered.

Distance in throw test performance is reported in table 2 as mean (SD) pre-exercise and postexercise maximum throw, total distance for all throws (pre-exercise and postexercise), \% decline in respective tests and total \% decline from pre-exercise

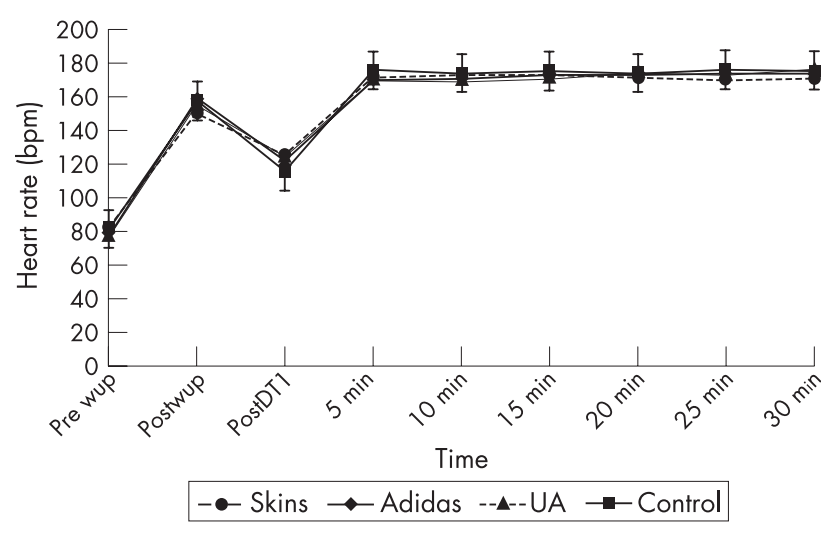

Figure 2 Mean heart rate response throughout the exercise protocol for Skins, Adidas, Under Armour (UA) and control conditions.

to post-exercise for all conditions. Analysis indicated no significant differences $(p>0.05)$ and small ES between all conditions for all maximal throwing performance measures, apart from a moderate-to-large ES for the total \% decline for the Adidas condition.

Figure 1 outlines AT test results for all conditions, including the mean scores for individual throws, total score and time to complete all throws for the pre-, $10 \mathrm{~min}, 20 \mathrm{~min}$ and $30 \mathrm{~min}$ AT test. Analysis indicated no significant differences $(p>0.05)$ for the accuracy score of individual throws, total accuracy score or time to complete AT test between conditions. ES analysis indicated a moderate effect of improved throw accuracy performance in the UA condition at 10 and $20 \mathrm{~min}$.

Table 3 presents the results for mean (SD) heart rate, predifference to postdifference in body mass, $\mathrm{T}_{\mathrm{sk}}$, postexercise RPE, average RPE, postexercise and $24 \mathrm{~h}$ postexercise rating of $\mathrm{MS}_{\mathrm{A}}$ and $\mathrm{MS}_{\mathrm{L}}$, respectively. Analysis indicated no significant differences $(p>0.05)$ and small ES between conditions for mean heart rate (fig 2), difference in mass or RPE measures. A significantly lower $\mathrm{T}_{\mathrm{sk}}(\mathrm{p}<0.05$ and large ES) was observed in the control condition compared with the respective compression garment conditions (fig 3). A significantly higher rating of $\mathrm{MS}_{\mathrm{A}}$ and $\mathrm{MS}_{\mathrm{L}} 24 \mathrm{~h}$ after exercise $(\mathrm{p}<0.05$ and large ES) was reported in the control condition compared with the three garment conditions, with no significant difference between the garment conditions. All pre-exercise ratings of MS had returned to 0 for all conditions.

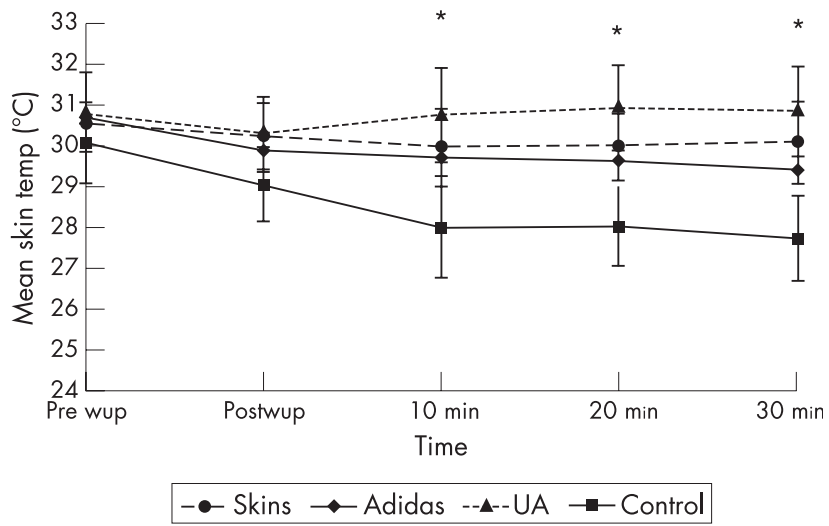

Figure 3 Mean skin temperature throughout the exercise protocol for Skins, Adidas, Under Armour (UA) and control conditions in a cool environment. *Compression garment conditions significantly different from control condition $(\mathrm{p}<0.05)$. 
Table 3 Mean (SD) heart rate, difference in body mass, mean skin temperature, rating of perceived exertion (RPE) at $30 \mathrm{~min}$, average exercise RPE, rating of muscle soreness for legs and arms post-testing and $24 \mathrm{~h}$ postexercise

\begin{tabular}{lllll}
\hline & Skins & Adidas & UA & Control \\
\hline HR (bpm) & $171(9)$ & $172(11)$ & $172(8)$ & $175(10)$ \\
Mass difference $(\mathrm{kg})$ & $0.76(0.13)$ & $0.71(0.13)$ & $0.71(0.13)$ & $0.75(0.19)$ \\
$\mathrm{T}_{\text {sk }}$ ('C) & $30.2(0.6)^{*}$ & $29.9(0.8)^{*}$ & $30.7(0.9)^{*}$ & $28.5(0.8)$ \\
RPE postexercise & $7.2(1.2)$ & $7.1(1.2)$ & $7.2(0.8)$ & $7.0(1.3)$ \\
RPE average & $5.8(1.2)$ & $5.6(1.2)$ & $5.6(0.8)$ & $5.6(1.5)$ \\
MS & $3.4(1.1)$ & $3.2(1.4)$ & $3.1(0.9)$ & $3.9(1.1)$ \\
$M_{L}$ 24texercise & $1.7(1.6)^{*}$ & $1.5(1.2)^{*}$ & $3.0(1.2)$ \\
$M_{\mathrm{A}}$ postexercexercise & $1.8(0.8)^{*}$ & $3.4(1.5)$ & $3.4(1.2)$ & $4.4(0.9)$ \\
$\mathrm{MS}_{\mathrm{A}}$ 24 h postexercise & $2.4 \pm 0.5^{*}$ & $2.5(1.3)^{*}$ & $1.7(1.1)^{*}$ & $3.4(1.1)$ \\
\hline
\end{tabular}

bpm, beats per minute; $H R$, heart rate; $M S_{A}$, muscle soreness for arms; $M S_{L}$, muscle soreness for legs; $R P E$, rating of perceived exertion; $T_{\text {sk, }}$ mean skin temperature; UA, Under Armour.

${ }^{*}$ Significantly different from control $(p<0.05)$

Table 4 presents the mean (SD) pre-exercise and postexercise capillary blood measures of $\left[\mathrm{La}^{-}\right], \mathrm{pH}, \mathrm{sO}_{2}, \mathrm{pO}_{2}$ and $\mathrm{CK}$. Analysis indicated no significant difference $(\mathrm{p}>0.05)$ and trivial ES between any condition for $\left[\mathrm{La}^{-}\right], \mathrm{pH}, \mathrm{sO}_{2}$ and $\mathrm{pO}_{2}$. Analysis of CK data indicated significantly lower $(\mathrm{p}<0.05)$ CK values $24 \mathrm{~h}$ after exercise in the Skins and UA conditions when compared with the control condition. Large ES data were observed for all three compression garment conditions when compared with the control condition, indicating that CK values were lower $24 \mathrm{~h}$ after exercise.

\section{DISCUSSION}

The aim of this study was to compare three varieties of compression garment (Skins, Adidas and UA) to determine whether repeat-sprint and throwing performance were improved. Results indicated neither throwing nor repeat-sprint performance was improved by any garment, and minimal differences were evident between garments. Significant physiological differences between control and compression garment conditions included higher $\mathrm{T}_{\mathrm{sk}}$ during exercise and reduced CK values $24 \mathrm{~h}$ after exercise in the compression garment conditions. Also, a significant difference was observed between compression garments and control conditions for the rating of $\mathrm{MS}_{\mathrm{A}}$ and $\mathrm{MS}_{\mathrm{L}} 24 \mathrm{~h}$ after exercise.

\section{Exercise performance}

No significant differences in 10 or $20 \mathrm{~m}$ sprint times, decline in sprint performance or submaximal distances covered because of or between garments were evident. Although currently there is no published research on the effects of compression garments on repeat-sprint performance, previous research has reported improved vertical jump heights without improvements in 20 or $60 \mathrm{~m}$ sprint time ${ }^{912}$ and increased force production in repeated vertical jump efforts ${ }^{13}$ while wearing compression garments. Improvements in maximal aerobic performance with compression garments have been reported in repeated 5 min maximal cycle efforts separated by an 80 min recovery. ${ }^{6}$ Recent research ${ }^{14}$ on fatigue recovery reported that compression garments did not increase fatigability during ankle dorsiflexion and did not improve force recovery between repeated fatiguing static efforts. In contrast, Kraemer et al ${ }^{15}$ reported a faster recovery of force production in single-arm bicep curls following heavy eccentric exercise when wearing compression garments. Whereas previous data have reported a variety of performance outcomes, few studies have investigated sports-specific performance benefits from compression garments, and no studies have reported improvements in repeat-sprint exercise. As such, the results of the present data show that compression garments did not improve repeat-sprint activity, reduce the decline in sprint performance or increase the distance covered, and may have limited ergogenic properties for repeat-sprint performance.

\section{Throwing performance}

Currently, there is no evidence of the influence of compression garments on throwing performance in literature. Although the garments have been shown to increase vertical jump, ${ }^{9}{ }^{13}$ there was no improvement in maximal throw distance or total distance, and only a small amelioration of the decline in maximal throw distance was observed solely in the Adidas garment condition. Further, apart from the Adidas \% decline shown in the ES data, only small ESs were noted between the

Table 4 Mean (SD) pre-exercise and postexercise measures of blood lactate concentration, pH, oxygen saturation of haemoglobin partial pressure of oxygen and pre-exercise and $24 \mathrm{~h}$ postexercise and \% change in creatine kinase

\begin{tabular}{|c|c|c|c|c|}
\hline & Skins & Adidas & UA & Control \\
\hline$\left[\mathrm{La}^{-}\right]_{\mathrm{b}}$ pre-exercise & $1.4(0.4)$ & $1.7(0.5)$ & $1.3(0.5)$ & $1.4(0.5)$ \\
\hline$\left[\mathrm{La}^{-}\right]_{\mathrm{b}}$ postexercise & $8.1(2.2)$ & $7.9(1.7)$ & $8.7(3.3)$ & $9.0(2.4)$ \\
\hline $\mathrm{pH}$ pre-exercise & $7.424(0.119)$ & $7.416(0.159)$ & $7.408(0.109)$ & $7.414(0.156)$ \\
\hline $\mathrm{pH}$ postexercise & $7.364(0.031)$ & $7.355(0.045)$ & $7.352(0.476)$ & $7.349(0.051)$ \\
\hline $\mathrm{sO}_{2}$ pre-exercise & $96.0(0.8)$ & $95.9(1.3)$ & $95.7(0.87)$ & $95.9(1.2)$ \\
\hline $\mathrm{sO}_{2}$ postexercise & $96.1(1.2)$ & $96.1(0.8)$ & $95.9(0.8)$ & $96.3(1.3)$ \\
\hline $\mathrm{pO}_{2}$ pre-exercise & $73.5(5.5)$ & $74.9(6.5)$ & $73.1(5.9)$ & $73.8(5.9)$ \\
\hline $\mathrm{pO}_{2}$ postexercise & $81.3(7.5)$ & $82.1(4.8)$ & $80.8(5.6)$ & $82.6(4.7)$ \\
\hline CK pre-exercise & 450 (189) & 493 (182) & 422 (125) & $518(145)$ \\
\hline CK $24 \mathrm{~h}$ postexercise & $1014(178)^{*} \dagger$ & $1105(192) \dagger$ & $1031(129)^{*} \dagger$ & $1266(120)$ \\
\hline CK \% change & $225(71)$ & $224(75)$ & $220(70)$ & $245(68)$ \\
\hline
\end{tabular}

$\mathrm{CK}$, creatine kinase; $\left[\mathrm{La}^{-}\right] \mathrm{b}$, blood lactate; $\mathrm{pO}_{2}$, partial pressure of oxygen; $\mathrm{s}_{2}$, oxygen saturation of haemoglobin; UA, Under Armour.

*Significantly different from control $(p<0.05)$.

†Large effect size compared with control $(d>0.8)$. 
conditions for singular or repeated throwing ability. Although Doan $\mathrm{et}^{\mathrm{al}}$ have reported that increased flexion and extension torque may result in a greater power output of the specific muscular action, no differences in distance thrown were evident in this study. Further, no significant improvements were noted for throwing accuracy or time to complete throwing activities during the testing protocol. Moderate ESs were evident to indicate an improved throw accuracy performance in the UA condition at 10 and $20 \mathrm{~min}$; however, any mechanisms to explain this difference, such as proprioreceptive feedback or increased flexion-extension torque, ${ }^{9}$ would be speculative.

\section{Physiological variables}

No significant difference in the heart rate response during the exercise protocol was evident in the current study. Berry et a $l^{16}$ have also reported no effect of compression garments on heart rate during an exhaustive run at $110 \% \mathrm{VO}_{2 \max }$. Several studies have reported the benefits of compression garments in reducing venous oedema, ${ }^{17}$ vascular pooling ${ }^{5}$ and enhancing overall circulation. ${ }^{7}$ However, Kahn et $a l^{18}$ have reported no changes in leg volume in patients with deep vein thrombosis following exercise in compression stockings, whereas Chatard et $a l^{6}$ reported small, non-significant increases in plasma volume from wearing compression garments during maximal $5 \mathrm{~min}$ efforts. In the current study, as heart rate did not differ, and as it is assumed cardiac output was unchanged between conditions, it is unlikely that any differences in venous return or stroke volume were evident between conditions.

Compression garments did not significantly alter the pre to post loss of body mass, indicating that sweat volume was similar between conditions. Similar sweat rates between the conditions (in cool to moderate environmental conditions) imply that no impedance to the sweating mechanism was evident during exercise. Whether the garments may have affected the efficient removal of body heat is unknown, as core temperature was not measured. However, in these mild environments, the effectiveness of conduction and convection is improved; hence any reduction in evaporative mechanisms is of less importance. $\mathrm{T}_{\mathrm{sk}}$ was significantly lower in the control condition, indicating a warmer skin temperature using all garments (with no significant differences between garments). A higher $\mathrm{T}_{\mathrm{sk}}$ without an increase in body mass loss in the compression garment conditions implies that effective thermoregulatory function was maintained over the 30 min exercise protocol. Doan et al have also reported a faster and greater rise in skin temperature under compression garments during a warm-up. As the current testing was conducted in cool environmental conditions, a higher $\mathrm{T}_{\text {sk }}$ would not unduly affect physiological functioning; however, exercise for longer durations and in warmer conditions may impose greater physiological strain and affect physiological functioning and/or performance.

Although several studies have reported reductions in $\left[\mathrm{La}^{-}\right]$ following exercise in compression garments, ${ }^{6}{ }^{19}$ in the current study no significant differences were observed between conditions for $\left[\mathrm{La}^{-}\right]$. Berry and McMurray ${ }^{19}$ observed reduced $\left[\mathrm{La}^{-}\right]$ during the recovery from a treadmill $\mathrm{VO}_{2 \max }$ (maximum amount of oxygen in milliliters, one can use in one minute per kilogram of body weight) test, also supported by Chatard et $a l^{6}$ following $5 \mathrm{~min}$ maximal efforts and an $80 \mathrm{~min}$ recovery. Conversely, Berry et al ${ }^{16}$ reported no significant effect of the garments in reducing $\left[\mathrm{La}^{-}\right]$following a run to exhaustion at $110 \% \mathrm{VO}_{2 \max }$. Both studies reporting changes in $\left[\mathrm{La}^{-}\right]$have also reported small plasma volume shifts, which may account for the observed reductions in $\left[\mathrm{La}^{-}\right]$. There were also no significant differences and small ESs for $\mathrm{pH}, \mathrm{sO}_{2}$ and $\mathrm{pO}_{2}$.
Trennell et $a l^{20}$ have reported no difference in muscle $\mathrm{pH}$ between control- and compression garment-covered limbs using ${ }^{31} \mathrm{p}$-MNR spectroscopy during eccentric downhill treadmill walking, while Agu et al reported an increase in limb oxygenation in patients with venous insufficiency through near infrared spectroscopy. Although compression garments may increase blood volume, and therefore $\mathrm{sO}_{2}$, in diseased patients, they did not increase haemoglobin saturation in healthy males. It seems unlikely that compression garments increase $\mathrm{sO}_{2}$ during maximal exercise, as evidenced by Berry et al, ${ }^{16}$ who reported no increase in $\mathrm{VO}_{2 \max }$ during maximal exercise while wearing compression garments.

A significantly reduced absolute CK value was observed in the Skins and UA conditions, and large ESs for all garments when compared with the control condition. Gill et $a l^{8}$ and Kraemer $e t$ al $^{15}$ have both reported lower CK values when using compression garments as opposed to normal clothing following high-intensity exercise. Both studies have proposed that the compression process acts to reduce swelling and limit the inflammatory response to acute muscle damage. As such, the act of applying compression via the garments in the $24 \mathrm{~h}$ following exercise may limit any swelling mechanisms resultant from microtrauma to the muscle. However, while exercise was avoided during the $24 \mathrm{~h}$ after testing, fluid and nutritional intakes were not controlled; yet the results of the \% change in CK showed no significant differences and small to moderate ES between conditions. These limitations to the data preclude any conclusive statements on the potential recovery benefits of wearing compression garments after exercise.

\section{Perception of effort and muscle soreness}

Although differences between conditions on most physiological measures were trivial, performance improvements may have resulted from changes in the perception of fatigue. In the present study, compression garments did not significantly reduce the mean or final RPE, and no differences were observed between the respective garments. However, significantly reduced (improved) ratings of $\mathrm{MS}_{\mathrm{A}}$ and $\mathrm{MS}_{\mathrm{L}}$ were reported by participants $24 \mathrm{~h}$ after exercise in compression garments, which fits with the lower CK values observed for the garments. These results indicate that the act of compression may help to improve subjective feelings of recovery when worn (including during sleep) after high-intensity exercise. Kraemer et $a l^{15}$ have also reported reductions in perceived muscle soreness with compression garments following heavy eccentric exercise. In contrast, Trennell et $a l^{20}$ reported no difference in

\section{What is already known on this topic}

Compression garments have been used in the medical industry for vascular patients, and their use has become popular with athletes; however, limited research exists on the ergogenic qualities for sports-specific exercise, with available research showing mixed results for exercise involving repeated powerful efforts.

\section{What this study adds}

This study indicates that compression garments did not improve repeat-sprint or repeated throwing performance; however, there may be benefits in their use as a thermal insulator in cool conditions, and further as a recovery intervention tool after high-intensity exercise. 
perceived muscle soreness with and without compression garments during or after downhill treadmill walking. As such, the use of compression garments may be of most benefit as a recovery aid to be worn during the $24-48 \mathrm{~h}$ after exercise to promote psychological recovery from high-intensity exercise, regardless of potential physiological changes.

In conclusion, compression garments did not improve throwing or repeat-sprint exercise performance in cricket players, with no differences in heart rate, body mass difference, blood measures of [ $\left.\mathrm{La}^{-}\right], \mathrm{pH}, \mathrm{sO}_{2}$ or $\mathrm{pO}_{2}$. Significant differences were observed by way of higher $\mathrm{T}_{\mathrm{sk}}$, lower $24 \mathrm{~h}$ postexercise CK values and lower $24 \mathrm{~h}$ postexercise ratings of muscle soreness in the compression garment conditions. In addition, there were only small differences between the three brands of compression garments. Overall, little performance benefit was noted when using the compression garments; however, compression garments may be beneficial as a thermal insulator in cool conditions or when long delays exist between exercise bouts. Further, the use of the garments as a recovery tool, when worn after intense exercise, may help reduce postexercise swelling and reduce perceived muscle soreness and promote greater psychological comfort.

\section{ACKNOWLEDGEMENTS}

The authors thank Cricket Australia for organising and funding this project and the respective companies, Skins, Adidas and Under Armour, for the generous supply of their products.

\section{Authors' affiliations}

Rob Duffield, School of Human Movement, Charles Stuart University, Bathurst, New South Wales, Australia

Marc Portus, Commonwealth Bank Centre of Excellence, Cricket Australia, Brisbane, Queensland, Australia

Competing interests: None declared.

\section{REFERENCES}

1 Lawrence D, Kakkar VV. Graduated, static, external compression of the lower limb: a physiological assessment. Br J Surg 1980;67:119-21.

2 Mayberry JC, Moneta GL, DeFrang RD, et al. The influence of elastic stockings on deep venous hemodynamics. J Vasc Surg 1991;13:91-9.

3 Wallace L, Slattery K, Coutts A. Compression garments: do they influence athletic performance and recovery? Sports Coach 2006;28:38-9.

4 lbegbuna V, Delis KT, Nicolaides AN, et al. Effect of elastic compression stockings on venous hemodynamics during walking. J Vasc Surg 2003;37:420-5
5 Agu O, Baker D, Seifalian AM. Effect of graduated compression stockings on limb oxygenation and venous function during exercise in patients with venous insufficiency. Vascular 2004; 12:69-76

6 Chatard JC, Atlaoui D, Farjanel J, et al. Elastic stockings, performance and leg pain recovery in 63-year-old sportsmen. Eur J Appl Physiol 2004;93:347-52.

7 Bringard A, Perrey S, Belluye N. Aerobic energy cost and sensation during submaximal running - positive effects of wearing compression tights. Int I Sports Med 2006;27:373-8

8 Gill ND, Beaven CM, Cook C. Effectiveness of post-match recovery strategies in rugby players. Br J Sports Med 2006:40:260-3.

9 Doan BK, Kwon YH, Newton RU, et al. Evaluation of a lower-body compression garment. J Sports Sci 2003;21:601-10.

10 Burton AC. New technique for the measurement of average skin temperature over surfaces of the body, and the changes of skin temperature during exercise. J Nutr 1934:7:48|-96.

11 Adams WC, Fox RH, Fry AJ, et al. Thermoregulation during marathon running in cool, moderate, and hot environments. J Appl Physiol 1975;38:1030-7.

12 Bernhardt T, Anderson GS. Influence of moderate prophylactic compression on sport performance. J Strength Cond Res 2005; 19:292-7.

13 Kraemer WJ, Bush JA, Baver JA, et al. Influence of compression garments on vertical jump performance in NCAA division I volleyball players. J Strength Cond Res 1996;10:180-3.

14 Maton B, Thieney G, Dang S, et al. Human muscle fatigue and elastic compressive stockings. Eur J Appl Physiol 2006:97:432-42.

15 Kraemer WJ, Bush JA, Wickham RB, et al. Influence of compression therapy on symptoms following soft tissue injury from maximal eccentric exercise. J Orthop Sports Phys Ther 2001;31:282-90

16 Berry MJ, Bailey SP, Simpkins LS, et al. The effect of elastic tights on the postexercise response. Can J Sport Sci 1990;15:144-8.

17 Vayssairat M, Ziani E, Houot B. Placebo controlled efficacy of class 1 elastic stockings in chronic venous insufficiency of the lower limb. J Mal Vasc 2000;25:256-62

18 Kahn SR, Azoulay L, Haber M, et al. Effect of graduated elastic compression stockings on leg symptoms and signs during exercise in patients with deep venous thrombosis: a randomized cross-over trial. J Thromb Haemost 2003;1:494-9.

19 Berry MJ, McMurray RG. Effects of graduated compression stockings on blood lactate following an exhaustive bout of exercise. Am J Phys Med 1987:66:121-32

20 Trennell MI, Rooney KB, Sue CM, et al. Compression garments and recovery from eccentric exercise: a ${ }^{31}$ P-MRS study. J Sports Sci Med 2006;5:106-14

\section{COMMENTARY}

This article shows that although compression garments have become popular within the sporting fraternity, the benefits may be more psychological, rather than physiological, and more research is required.

Johann Edge

Massey University, Palmerston North, New Zealand; i.a.edge@massey.ac.nz

Submit an eLetter, and join the debate

eLetters are a fast and convenient way to register your opinion on topical and contentious medical issues. You can find the "submit a response" link alongside the abstract, full text and PDF versions of all our articles. We aim to publish swiftly, and your comments will be emailed directly to the author of the original article to allow them to respond. eletters are a great way of participating in important clinical debates, so make sure your voice is heard. 